\title{
Negligible Effect of Perioperative Epidural Analgesia Among Patients Undergoing Elective Gastric and Pancreatic Resections
}

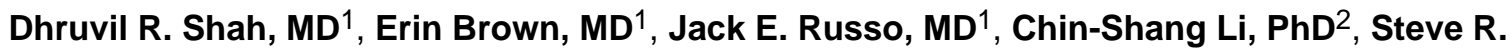 \\ Martinez, MD, MAS ${ }^{1}$, Jodi M. Coates, MD ${ }^{1}$, Richard J. Bold, MD ${ }^{1}$, and Robert J. Canter, MD ${ }^{1}$ \\ ${ }^{1}$ Division of Surgical Oncology, UC Davis Cancer Center, Sacramento, CA 95817 \\ ${ }^{2}$ Division of Biostatistics, Department of Public Health Sciences, University of California Davis, \\ Davis, CA 95616
}

\section{Abstract}

Background-There are conflicting data regarding improvements in postoperative outcomes with perioperative epidural analgesia. We sought to examine the effect of perioperative epidural analgesia versus intravenous narcotic analgesia on perioperative outcomes including pain control, morbidity, and mortality in patients undergoing gastric and pancreatic resections.

\begin{abstract}
Methods-We evaluated 169 patients from 2007 to 2011 who underwent open gastric and pancreatic resections for malignancy at a university medical center. Emergency, traumatic, pediatric, enucleations, and disseminated cancer cases were excluded. Clinicopathologic data were reviewed among epidural (E) and non-epidural (NE) patients for their association with perioperative endpoints.
\end{abstract}

Results-120 patients (71\%) received an epidural, and 49 (29\%) did not. There were no significant differences $(\mathrm{P}>0.05)$ in mean pain scores at each of the four days (days 0-3) among $\mathrm{E}$ ( $3.2 \pm 2.7,3.2 \pm 2.3,2.3 \pm 1.9$, and $2.1 \pm 1.9$, respectively) and NE patients ( $3.7 \pm 2.7,3.4 \pm 1.9$, $2.9 \pm 2.1$, and $2.4 \pm 1.9$, respectively). Within each of the $\mathrm{E}$ and NE patient groups, there were significant differences $(\mathrm{P}<0.0001)$ in mean pain scores from day 0 to day $3(\mathrm{P}<0.0001) .69 \%$ of $\mathrm{E}$ patients also received intravenous patient-controlled analgesia (PCA). Ileus (13\% E vs. $8 \% \mathrm{NE}$ ), pneumonia $(12 \% \mathrm{E}$ vs. $8 \% \mathrm{NE})$, venous thromboembolism ( $6 \% \mathrm{E}$ vs. $4 \% \mathrm{NE})$, length of stay [ $11.0 \pm 12.1(8,4-107)$ E vs. $12.2 \pm 10.7(7,3-54) \mathrm{NE}$ ], overall morbidity ( $36 \% \mathrm{E}$ vs. $39 \% \mathrm{NE})$, and mortality ( $4 \% \mathrm{E}$ vs. $2 \% \mathrm{NE})$ were not significantly different.

Conclusions-Routine use of epidurals in this group of patients does not appear to be superior to PCA.

\section{Keywords}

epidural analgesia; upper gastrointestinal surgery; morbidity; mortality

Correspondence: Robert J. Canter, MD Division of Surgical Oncology, Suite 3010 UC Davis Cancer Center 4501 X Street Sacramento, CA 95817 Phone: (916) 734-7044 Fax: (916) 703-5267 Robert.canter@ucdmc.ucdavis.edu. 


\section{Introduction}

Perioperative epidural analgesia is regularly used in major abdominal operations. Proponents of this modality point to studies demonstrating modest improvements in postoperative pain control with epidurals as compared to intravenous analgesics [1,2]. Studies of epidural analgesia after abdominal surgery have also reported decreased rates of ileus [3,4], pneumonia [5], venous thromboembolism, and immunosuppression [6] among epidural patients when compared to patients managed without epidurals. However, meta-analyses and randomized control trials which have analyzed perioperative outcomes of epidurals following laparotomy have reported conflicting data $[7,8]$, raising questions regarding the purported benefits of epidurals. Moreover, recent studies have highlighted the resourceintensive nature of the placement of epidural catheters [9] as well as the potential for procedural complications such as hypotension and premature catheter dislodgement which occur in the range of $14-40 \%[3,10]$.

Given these conflicting data, we sought to analyze the perioperative outcomes of contemporaneous cohorts of patients undergoing major upper gastrointestinal surgery (gastric and pancreatic resections) to determine if significant differences in outcome exist between patients receiving epidural analgesia versus those who received alternate forms of postoperative pain control. We hypothesized that the purported benefits of epidural analgesia would not extend to patients undergoing major upper gastrointestinal tumor resections.

\section{Methods}

From January 2007 to June 2011, 169 patients underwent elective gastric and pancreatic resections for space-occupying lesions at a single-institution university tertiary referral center. Emergency, traumatic, pediatric, and disseminated cancer cases were excluded. Nonanatomic tumor resections such as enucleations were also excluded.

Decisions regarding use of epidural analgesia for postoperative pain control were based on surgeon, anesthesiologist, and patient preference. All epidural catheters were placed preoperatively by the resident and attending anesthesiologist conducting the anesthetic management for the case. The epidural was placed between the T9 and T12 interspaces depending on patient anatomy and anesthesiologist preference. Successful placement of the epidural was confirmed with a test dose of $1 \%$ lidocaine providing anesthesia to the appropriate dermatome region without pain or paresthesias. All epidural catheters were assessed and adjusted on a daily basis post-operatively by a dedicated Acute Pain consult service supervised by a faculty anesthesiologist. Catheters were infused with $0.25 \%$ bupivicaine and either hydromorphone or morphine as a continuous infusion with the rate of infusion varying based on clinical parameters such as pain control and hemodynamic response to the infusion. Boluses of analgesia were administered at the discretion of the Acute Pain consult service.

In cases of inadequate analgesia, medication adjustments were made in the epidural infusion. However, patients were also frequently placed on concomitant PCA infusion with 
narcotics while infusing local anesthetic into the epidural. These decisions were also made at the discretion of the Acute Pain consult service, although input from the surgical service was often a significant factor in these decisions. Among NE patients, breakthrough pain was managed by the primary surgical team and included increased doses of narcotics through the PCA, supplemental IV narcotic doses, and/or ketorolac. No patient in this study received intravenous Tylenol. Among patients receiving oral alimentation, breakthrough pain was occasionally managed with oral narcotics if oral medications had been initiated and were tolerated. No NE patients received an epidural catheter for breakthrough pain.

Following approval for this study by the Institutional Review Board, clinicopathologic data were reviewed among epidural (E) and non-epidural (NE) patients for their association with perioperative outcomes. Data were collected on age, sex, diagnosis, American Society for Anesthesiology (ASA) physical status class, surgery performed including adjacent organ resection, type of incision, length of surgery, and estimated blood loss. Length of surgery was strictly defined as time from incision to closure. Time spent in the operating room on epidural placement and induction of anesthesia was excluded. Multivisceral resection was defined as any additional organ resection not considered part of the primary procedure. Sufficient data on BMI were not available to permit analysis of this predictor variable.

Numerical postoperative pain scores $(0-10)$ for each individual patient were obtained from the electronic medical record as documented in the nursing assessment forms. These were recorded for patients based on standardized protocols set forth by the medical center and the Acute Pain service. All recorded values for a given day were abstracted, starting on the day of surgery [postoperative day (POD) zero] and continuing until POD three. The majority of epidural catheters were discontinued between POD 3 and 5 as patients were transitioned to oral analgesics. Individual pain scores and number of observations obtained per patient per postoperative day were recorded. For a patient who had multiple pain assessments at a given day, the sample mean of the multiple pain scores was used a pain score at the given day for an analysis of repeated measures data. Patients were assessed for the duration of epidural use, and patients who underwent removal of their epidural catheter within 48 hours of the index operation were considered to have an ineffective epidural.

Complications were ascertained by retrospective review of the medical record using standardized definitions [11]. We defined pneumonia based on the 2005 American Thoracic Society [12] guidelines as a condition characterized by (1) an infiltrate on chest X-ray along with (2) clinical findings suggesting infection such as purulent secretions, fever, leukocytosis, or increasing oxygen requirements and (3) the institution of antibiotic therapy based on the presence of either of the above findings. Gastro-intestinal leak/abscess/fistula was defined as an intra-abdominal fluid collection requiring drainage and/or any measurable amount of drain fluid after postoperative day 3 with an amylase content greater than 3 times the serum amylase for pancreatic resections [13]. Postoperative ileus was defined as nausea, vomiting, or inability to tolerate oral intake by POD ten. Given the variable definitions of this endpoint in the literature, we chose to analyze a longer threshold for delayed return of bowel function based on previous studies of delayed gastric emptying and prolonged ileus following pancreaticoduodenectomy [14]. Venous thromboembolism (VTE) was defined as 
either an acute pulmonary embolism diagnosed on chest $\mathrm{CT}$ or an acute proximal lower extremity deep vein thrombosis diagnosed with duplex ultrasound.

Summary statistics of a numerical variable were expressed as mean \pm standard deviation (median, minimum - maximum). Categorical variables were compared using Fisher's exact test. A numerical variable was compared between two groups by using two-sided twosample t-test or Wilcoxon ran-sum test when appropriate. Multivariable logistic regression was used to identify significant predictors of effective analgesia with epidural monotherapy among patients not receiving a PCA or IV narcotics while the epidural was in place. We reported odds ratios (ORs) and 95\% confidence intervals (95\% CIs) to identify significant associations. All statistical analyses were performed with SAS v9.2 (Cary, NC, USA). .

\section{Results}

\section{Clinicopathologic and Treatment Characteristics}

There were 120 patients (71\%) who received an epidural (E), and 49 (29\%) who did not (NE). Table 1 depicts the clinicopathologic characteristics of the two cohorts of patients. Age, proportion of men and women, proportion of ASA class 3, and median operative blood loss were statistically similar among $\mathrm{E}$ and NE patients. In contrast, among E patients, there was a greater proportion of patients diagnosed with pancreatic cancer $(59 \%$ vs. $22 \%, \mathrm{P}<$ 0.0001 ) and patients undergoing pancreatectomy ( $90 \%$ vs. $51 \%, \mathrm{P}<0.0001)$. There were also significant differences among the $\mathrm{E}$ and $\mathrm{NE}$ patients regarding the incision used. The majority of E patients had a subcostal incision (83\%), while the majority of the NE patients had a midline incision $(76 \%, \mathrm{P}<0.0001)$. Operative time was also significantly different $[331 \pm 101(399,131$ - 705) $\mathrm{min}$ for E patients compared to $269 \pm 90(263,90$ - 498) min for NE patients, $\mathrm{P}=0.0001]$. A smaller proportion of $\mathrm{E}$ patients underwent multivisceral resection ( $12 \%$ vs. $27 \%, \mathrm{P}=0.02)$.

\section{Pain Scores}

As depicted in Figure 1, a repeated measures analysis of variance indicates that there was no significant difference in the pain scores between the $\mathrm{E}$ and NE cohorts at each of POD 0 3., Both E and NE patients demonstrated a similar trend of decreasing pain scores with each successive day, and this trend was statistically significant within each patient group $(\mathrm{P}<$ 0.0001). The median numbers of pain assessments per day per patient were also similar among $\mathrm{E}$ and NE patients, with the sole exception of POD 0 when NE patients had fewer pain assessments as the E patients. The differences in number of pain assessments on POD 0 were likely related to time of day the procedure was completed as well as post-anesthesia care unit nursing protocols. Subgroup analysis by procedure type (proximal pancreatectomy, distal pancreatectomy, and partial gastrectomy) revealed no differences in pain scores at each of POD $0-3$. Subgroup analysis of patients with an effective epidural (i.e. those whose epidural catheters were not prematurely discontinued or required concomitant PCA use) revealed incremental improvements in pain scores over NE patients, but these differences were not statistically significant. 


\section{Characteristics of Analgesic Use}

Nearly all of the patients in the NE group (96\%) used intravenous (IV) patient-controlled analgesia (PCA) with opioid medications for postoperative pain control (Table 2). However, a considerable number of patients (69\%) in the E group also used a PCA during the postoperative period. An additional $32 \%$ of the E group was administered repeated supplemental IV narcotics for breakthrough pain while receiving epidural analgesia (with or without a PCA). As depicted in Table 2, ketorolac was used in a similar proportion of $E$ and NE patients (24\% and $24 \%, \mathrm{P}=1.00$ ). Thirty-three patients (28\%) had their epidurals removed within two days of their operation. The reasons for early epidural discontinuation included inadvertent catheter dislodgement, hypotension, and/or inadequate pain control.

\section{Multivariable Analysis}

In order to assess the possibility for selective epidural catheter placement, we performed multivariable analysis on all E patients to identify the subset of patients in whom epidural catheters provided effective analgesia as monotherapy. As depicted in Table 3, neither age, sex, ASA class, pathologic diagnosis, type of operation performed, incision type, or performance of multivisceral resection significantly predicted effective analgesia with epidural monotherapy.

\section{Perioperative Outcomes, Morbidity, and Mortality}

As seen in Table 4, there were no significant differences in the median number of days until patients started a clear liquid diet [3.5 $\pm 2.6(3,1-25)$ E vs. $3.1 \pm 2.1(2,1-10) \mathrm{NE}, \mathrm{P}=0.07]$ or a solid diet $[5.8 \pm 3.3(5,2-28)$ E vs. $7.0 \pm 5.8(5,2-31) \mathrm{NE}, \mathrm{P}=0.58]$. The median hospital length

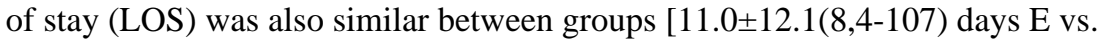
$12.2 \pm 10.7(7,3-54)$ days $\mathrm{NE}, \mathrm{P}=0.93$ ]. In addition, rates of pneumonia (12\% $\mathrm{E}$ vs. $8 \% \mathrm{NE}$, $\mathrm{P}=0.59)$, deep vein thrombosis or pulmonary embolism ( $6 \%$ E vs. $4 \% \mathrm{NE}, \mathrm{P}=1.00)$, postoperative ileus ( $13 \% \mathrm{E}$ vs. $8 \% \mathrm{NE}, \mathrm{P}=0.44$ ), and overall morbidity (36\% E. vs. $39 \% \mathrm{NE}$, $\mathrm{P}=0.73$ ) were comparable between the cohorts. There was a statistically significantly decreased proportion of patients who developed a pancreatic leak in the Epidural group ( $10 \% \mathrm{E}$ vs. $28 \% \mathrm{NE}, \mathrm{P}=0.045$ ). Although there were five (4\%) post-operative deaths in the $\mathrm{E}$ group compared to one (2\%) patient in the NE group, this difference was not statistically significant $(\mathrm{P}=0.67)$. All postoperative mortalities occurred in patients who had undergone partial pancreatectomy.

Since the development of postoperative complications may potentially bias pain scores as well as predispose to the occurrence of secondary complications, we performed a subgroup analysis among patients who were free from postoperative complications. This subgroup analysis demonstrated no differences in pain scores, time to clear diet, time to regular diet, or LOS (data not shown). In addition, given the high rate of poorly functioning catheters (early discontinuation or inadequate pain relief) in our study, we performed a separate subgroup analysis comparing outcomes between patients who had an effective epidural catheter (those which were not prematurely discontinued or required concomitant PCA use) and those without an epidural catheter. As depicted in Table 4b, we observed no difference in perioperative morbidity or length of stay. 


\section{Discussion}

Although some authors consider perioperative epidural analgesia to be a standard analgesic adjunct for patients undergoing laparotomy, the data supporting their routine use, including those from randomized controlled trials and meta-analyses, are equivocal $[1,15])$. While studies performed more than a decade ago reported significant improvements in perioperative outcomes and overall morbidity with epidurals, contemporary studies have not. For example, a meta-analysis performed on studies prior to 1997 by Rodgers et al [8] reported a 33\% reduction in overall mortality and significant reductions in venous thromboembolism, pneumonia, myocardial infarction and renal failure with epidurals. However, a more recent meta-analysis performed by Werawatganon et al [1] was unable to find sufficient evidence to draw a similar conclusion. This difference may be attributable to a combination of increasing use of patient-controlled analgesia, improved analgesic adjuncts such as ketorolac, and reduction in overall surgical risk [5].

Our results suggest that epidural anesthesia when compared to IV PCA is not superior in reducing postoperative pain, ileus, morbidity, mortality, or LOS among patients undergoing gastric and pancreatic resections. Other recent large randomized trials by Park et al. [15] and the MASTER trial by Rigg et al. [7] support these results. Other studies have also suggested that the potential benefits of epidurals are operation specific. For example, randomized controlled trials in patients undergoing bowel resections for colorectal cancer have demonstrated no benefit to epidural analgesia in improving perioperative outcomes[3,16-18]. Our study is the first to examine the impact of epidural analgesia in patients undergoing complex, non-vascular upper abdominal operations such as gastric and pancreatic resections. Since these operations have higher associated morbidity and mortality, it is conceivable that epidural analgesia may have a different effect on postoperative pain and post-surgical morbidity.

An important potential limitation of our study is the expertise and experience of the providers performing the epidural placement. We observed that approximately one-third of E patients had poorly positioned or non-functioning pain catheters. Moreover, $28 \%$ of epidural catheters were removed within 48 hours of placement because of inadvertent catheter dislodgement, hypotension, or inadequate pain control. It is possible that the results we observed with epidural catheters are inferior to those obtained at other centers, thereby limiting the ability to extrapolate our data. However, large studies on the use of perioperative epidural analgesia have reported similar early failure rates [7], and it is debatable if the resources necessary to optimize the efficacy of epidurals can translate into clinically relevant and cost-effective benefits to patients.

Selective epidural catheter placement in patients who are most likely to derive a benefit in pain improvement would help maximize the cost-effectiveness of epidurals. We attempted to identify these subset of patients through multivariate analysis; however neither of the patient demographic or operative factors we examined were significant predictors. Other baseline clinical characteristics which were not available to us but could potentially impact the effectiveness of epidural analgesia as monotherapy include patient history of chronic narcotic use or chronic pain. However, a decreased efficacy of epidural blockade in patients 
with chronic pain in not well established, and the limited data which are available do not demonstrate a strong correlation between the degree of post-operative pain and a baseline history of chronic pain [19].

Since this is a retrospective analysis, patients were not randomly assigned to epidural or non-epidural analgesia. Therefore, we acknowledge the potential for selection bias in our study which may have led to an imbalance of important baseline characteristics between the cohorts. This imbalance could confound our outcome measures, such as pain scores or purported narcotic-related postoperative events such as ileus. For example, bilateral or extended subcostal incisions were significantly more common among E patients than NE patients. While the degree of pain may be different depending on incision type, on multivariate analysis, the type of incision was not a significant predictor of adequate pain relief with an epidural catheter. Moreover, prior studies have not reliably established a difference in postoperative pain or pulmonary complications between subcostal and midline incisions [20], and a Cochrane review published in 2005 (Cochrane Database of Systemic Reviews, 2005, Issue 4. Art. No.: CD005199) concluded that the effect of incision type on pain scores was difficult to interpret and that transverse incisions may be "less painful" than midline ones.

We also recognize that pancreatic resections were more common among E patients than NE patients, and operative time was correspondingly longer, and that data on BMI were unavailable. Although it is conceivable that these differences may have predisposed the $\mathrm{E}$ cohort to greater postoperative pain thereby obscuring the potential benefit of epidurals, there are few data demonstrating a correlation between type of abdominal organ removed and the extent of postoperative pain. Although one would likely expect a higher morbidity and mortality rate among pancreatectomy patients compared to gastrectomy patients (assuming a comparable distribution of comorbid medical conditions), there is no evidence in our data that epidurals reduced these rates, particularly for purported narcotic/pain-related outcomes such as ileus, pneumonia, and VTE.

Finally, it is possible that with a larger study population the observed differences between the groups may acquire statistical significance, suggesting that we may not have sufficient power to detect small differences in mean postoperative pain scores or other outcomes. Alternatively, even with greater numbers of patients, the differences in pain scores and morbidity rates may remain negligible, confirming a lack of benefit of epidural analgesia among patients undergoing elective gastric and pancreatic resections. In either case, our results do not suggest increased morbidity from omitting epidural analgesia. Despite the potential limitations of our study, we question the clinical significance of a presumed modest decrease in average postoperative pain scores, particularly if it does not correlate with improved perioperative outcomes or length of stay.

Given the implications of our study regarding resource utilization, we performed a limited analysis of costs and charges for a subset of 10 patients ( $6 \mathrm{E}$ and $4 \mathrm{NE}$ ). We identified charges of approximately $\$ 800$ for epidural placement with payments of roughly $\$ 186$, and we identified charges for epidural management of approximately $\$ 1,000$ to $\$ 1,250$ per day with payments of roughly $\$ 220$ to $\$ 350$ per day. Operating room time for these complex 
procedures loosely translates to $\$ 122$ per minute (therefore in the range of $\$ 3,050$ for a 25 minute epidural placement on average) and operating room charges for epidural medications ranges from $\$ 350$ to $\$ 400$, Although these figures appear to suggest that there may be significant cost and resource savings with a selective epidural approach, we also recognize that these calculations are likely to vary substantially depending on numerous factors such as institution, payer mix, and year of analysis, and a detailed cost-effectiveness analysis is beyond the scope of this study.

In summary, we provide a retrospective analysis of a contemporary cohort of patients undergoing elective gastric and pancreatic resections at a tertiary university referral center. We demonstrate that the use of epidural catheters does not alter pain scores or other postoperative outcomes among this patient population. This lack of improvement may be attributable to the impact of analgesic adjuncts such as patient-controlled analgesia and ketorolac or to ineffective pain control in a subset of patients with epidurals. Regardless, our results suggest that routine use of epidurals in this group of patients does not appear to be superior to PCA and the decision to use perioperative epidural analgesia should be guided by regular institution audits of their effectiveness.

\section{Acknowledgments}

Statistical services are supported by the National Center for Advancing Translational Sciences, National Institutes of Health, through grant \#UL1 TR000002.

\section{References}

1. Werawatganon TC, Somrat. Patient Controlled Intravenous opiod analgesia versus continous epidural analgesia for pain after intra-abdominal surgery (Review). The Cochrane Library. 2008:19.

2. Block BML, Spencer S, Bowlingson Andrew J. Cowan Anne R. Cowan John A. Wu Christopher L. Efficacy of Postoperative Epidural Analgesia - A Meta-analysis. JAMA. 2003; 290:9.

3. Marret E, Remy C, Bonnet F. Meta-analysis of epidural analgesia versus parenteral opioid analgesia after colorectal surgery. Br J Surg. 2007; 94:665-673. [PubMed: 17514701]

4. Jorgensen H, Wetterslev J, Moiniche S, Dahl JB. Epidural local anaesthetics versus opioid-based analgesic regimens on postoperative gastrointestinal paralysis, PONV and pain after abdominal surgery. Cochrane Database Syst Rev. 2000:CD001893. [PubMed: 11034732]

5. Popping DME, Nadia; Marret, Emmanuel; Remy, Camille; Tramer, Martine R. Protective Effects of Epidural Analgesia on Pulmonary Complications after Abdominal and Thoracic Surgery. Archives of Surgery. 2008; 143:10. [PubMed: 18209147]

6. Moselli NM, Baricocchi E, Ribero D, Sottile A, Suita L, Debernardi F. Intraoperative epidural analgesia prevents the early proinflammatory response to surgical trauma. Results from a prospective randomized clinical trial of intraoperative epidural versus general analgesia. Ann Surg Oncol. 2011; 18:2722-2731. [PubMed: 21479690]

7. Rigg JRA, Jamrozik K, Myles PS, Silbert BS, Peyton PJ, Parsons RW, Collins KS. Epidural anaesthesia and analgesia and outcome of major surgery: a randomised trial. The Lancet. 2002; 359:1276-1282.

8. Rodgers AW, Natalie; Schug, S.; McKee, A.; Kehlet, H.; van Zundert, A.; Sage, D.; Futter, M.; Saville, G.; Clark, T.; MacMahon, S. Reduction of postoperative mortality and morbidity with epidural or spinal anasthesia: results from overview of randomised trials. British Medical Journal. 2000; 321:12. [PubMed: 10875824]

9. Bartha E, Carlsson P, Kalman S. Evaluation of costs and effects of epidural analgesia and patientcontrolled intravenous analgesia after major abdominal surgery. Br J Anaesth. 2006; 96:111-117. [PubMed: 16257994] 
10. Konigsrainer I, Bredanger S, Drewel-Frohnmeyer R, Vonthein R, Krueger WA, Konigsrainer A, Unertl KE, Schroeder TH. Audit of motor weakness and premature catheter dislodgement after epidural analgesia in major abdominal surgery. Anaesthesia. 2009; 64:27-31. [PubMed: 18671685]

11. Grobmyer SR, Pieracci FM, Allen PJ, Brennan MF, Jaques DP. Defining morbidity after pancreaticoduodenectomy: use of a prospective complication grading system. J Am Coll Surg. 2007; 204:356-364. [PubMed: 17324768]

12. Guidelines for the management of adults with hospital-acquired, ventilator-associated, and healthcare-associated pneumonia. Am J Respir Crit Care Med. 2005; 171:388-416. [PubMed: 15699079]

13. Bassi C, Dervenis C, Butturini G, Fingerhut A, Yeo C, Izbicki J, Neoptolemos J, Sarr M, Traverso W, Buchler M. Postoperative pancreatic fistula: an international study group (ISGPF) definition. Surgery. 2005; 138:8-13. [PubMed: 16003309]

14. Balcom, JHt; Rattner, DW.; Warshaw, AL.; Chang, Y.; Fernandez-del Castillo, C. Ten-year experience with 733 pancreatic resections: changing indications, older patients, and decreasing length of hospitalization. Arch Surg. 2001; 136:391-398. [PubMed: 11296108]

15. Park WYT, Jon S, Lee Kelvin K. Effect of Epidural Anesthesia and Analgesia on Perioperative Outcome - A Randomized, Controlled Veternals Affairs Cooperative Study. Annals of Surgery. $2001 ; 234: 12$.

16. Zutshi M, Delaney CP, Senagore AJ, Mekhail N, Lewis B, Connor JT, Fazio VW. Randomized controlled trial comparing the controlled rehabilitation with early ambulation and diet pathway versus the controlled rehabilitation with early ambulation and diet with preemptive epidural anesthesia/analgesia after laparotomy and intestinal resection. Am J Surg. 2005; 189:268-272. [PubMed: 15792748]

17. Turunen P, Carpelan-Holmstrom M, Kairaluoma P, Wikstrom H, Kruuna O, Pere P, Bachmann M, Sarna S, Scheinin T. Epidural analgesia diminished pain but did not otherwise improve enhanced recovery after laparoscopic sigmoidectomy: a prospective randomized study. Surg Endosc. 2009; 23:31-37. [PubMed: 18814016]

18. Gendall KA, Kennedy RR, Watson AJM, Frizelle FA. The effect of epidural analgesia on postoperative outcome after colorectal surgery. Colorectal Disease. 2007; 9:584-598. [PubMed: 17506795]

19. Duncan F. Prospective observational study of postoperative epidural analgesia for major abdominal surgery. J Clin Nurs. 2011; 20:1870-1879. [PubMed: 21615577]

20. Seiler CM, Deckert A, Diener MK, Knaebel HP, Weigand MA, Victor N, Buchler MW. Midline versus transverse incision in major abdominal surgery: a randomized, double-blind equivalence trial (POVATI: ISRCTN60734227). Ann Surg. 2009; 249:913-920. [PubMed: 19474689] 


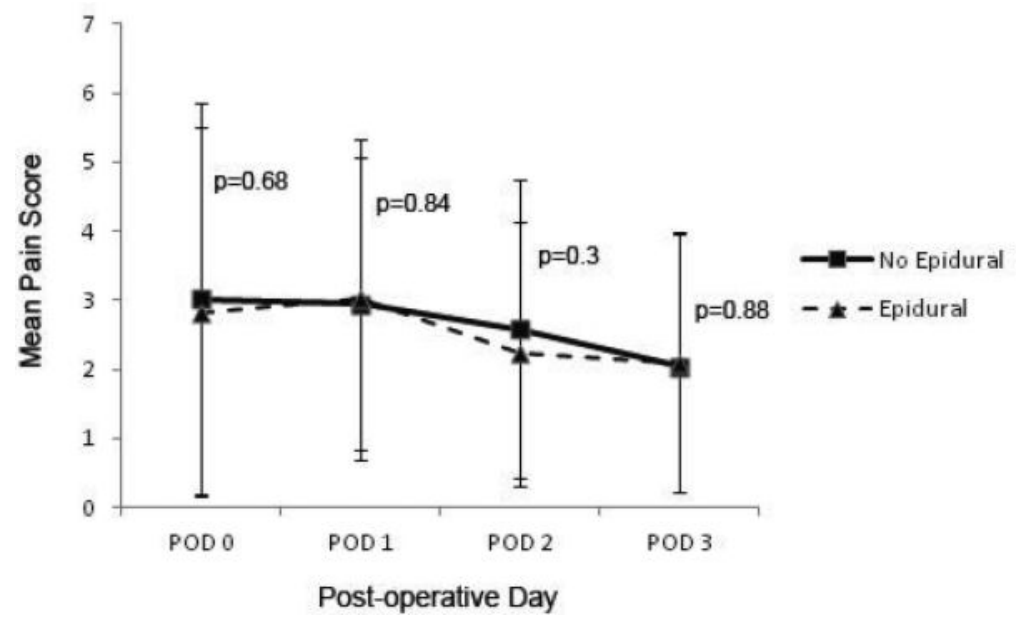

Figure 1.

Mean post-operative pain scores (scale 0-10) for Epidural and No Epidural patients. Error bars represent standard deviations. 
Table 1

Clinicopathologic and Treatment Characteristics

\begin{tabular}{|c|c|c|c|}
\hline & Epidural (N=120) & No Epidural (N=49) & P-Value \\
\hline Age (years) & $65 \pm 13.26(66,26-90)$ & $61 \pm 16.73(64,18-84)$ & 0.32 \\
\hline Sex & & & 0.18 \\
\hline Male & $63(52.5 \%)$ & $20(41 \%)$ & \\
\hline Female & $57(47.5 \%)$ & $29(59 \%)$ & \\
\hline ASA Class & & & 0.90 \\
\hline 2 & $31(26 \%)$ & $11(22 \%)$ & \\
\hline 3 & $88(73 \%)$ & $35(71 \%)$ & \\
\hline Other & $1(1 \%)$ & $3(7 \%)$ & \\
\hline Pathologic Diagnosis & & & $<0.0001$ \\
\hline Pancreatic Cancer & $71(59 \%)$ & $11(22 \%)$ & \\
\hline Gastric Cancer & $5(4 \%)$ & $14(29 \%)$ & \\
\hline Other Malignant & $31(26 \%)$ & $13(27 \%)$ & \\
\hline Benign & $13(11 \%)$ & $11(22 \%)$ & \\
\hline Surgical Procedure & & & $<0.0001$ \\
\hline Proximal Pancreatectomy & $87(73 \%)$ & $15(31 \%)$ & \\
\hline Distal Pancreatectomy & $20(17 \%)$ & $10(20 \%)$ & \\
\hline Partial Gastrectomy & $9(8 \%)$ & $18(37 \%)$ & \\
\hline Total Gastrectomy & $3(2 \%)$ & $6(12 \%)$ & \\
\hline Type of Incision & & & $<0.0001$ \\
\hline Subcostal & $100(83 \%)$ & $12(24 \%)$ & \\
\hline Midline & $20(17 \%)$ & $37(76 \%)$ & \\
\hline Length of Surgery (minutes) & $331 \pm 101(329,131-705)$ & $269 \pm 90(263,90-498)$ & 0.0001 \\
\hline $\mathbf{E B L}(\mathbf{m l})$ & $554 \pm 563(400,30-4900)$ & $815 \pm 1050(500,30-6000)$ & 0.14 \\
\hline Multivisceral Resection ${ }^{I}$ & $14(12 \%)$ & $13(27 \%)$ & 0.02 \\
\hline
\end{tabular}

Abbreviations: $\mathrm{E}=$ epidural, $\mathrm{NE}=$ No-epidural

${ }^{\text {II }}$ Defined as additional operation not part of primary procedure, including partial colectomy or partial hepatectomy. 
Table 2

Spectrum of Analgesic Use

\begin{tabular}{|l|l|l|l|}
\hline & Epidural(N=120) & No Epidural(N=49) & P-value \\
\hline PCA Use & $83(69 \%)$ & $47(96 \%)$ & - \\
\hline IV Narcotic Use with Epidural & $38(32 \%)$ & & - \\
\hline Epidural Discontinued in $\leq 2$ days & $33(28 \%)$ & & - \\
\hline Ketorolac Use & $29(24 \%)$ & $12(24 \%)$ & 1.00 \\
\hline
\end{tabular}

Abbreviations: PCA - Patient controlled analgesia; IV -intravenous 
Table 3

Multivariable Analysis of Predictors for Effective Epidural

\begin{tabular}{|l|l|l|l|}
\hline & OR & 95\% CI & P-Value \\
\hline Age & 1.006 & $0.971-1.041$ & 0.75 \\
\hline Sex & & & \\
\hline Female & Reference & Reference & \\
\hline Male & 0.833 & $0.382-1.819$ & 0.65 \\
\hline ASA Class & & & \\
\hline $1 \& 2$ & Reference & Reference & \\
\hline$\geq 3$ & 1.54 & $0.564-4.201$ & 0.40 \\
\hline Pathologic Diagnosis & & & \\
\hline Pancreatic Cancer & Reference & Reference & \\
\hline Benign & 0.175 & $0.01-2.982$ & 0.29 \\
\hline Gastric Cancer & 1.253 & $0.062-25.156$ & 0.88 \\
\hline Other Malignancy & 1.942 & $0.703-5.37$ & 0.20 \\
\hline Malignancy & & & \\
\hline No & Reference & Reference & \\
\hline Yes & 0.383 & $0.032-4.537$ & 0.45 \\
\hline Surgical Procedure & & & \\
\hline Pancreatectomy & Reference & Reference & \\
\hline Gastrectomy & 0.388 & $0.037-4.102$ & 0.43 \\
\hline Type of Incision & & & \\
\hline Subcostal & Reference & Reference & \\
\hline Midline & 0.913 & $0.185-4.501$ & 0.91 \\
\hline Multivisceral Resection & & & \\
\hline No & Reference & Reference & \\
\hline Yes & 1.039 & $0.3-3.593$ & 0.95 \\
\hline & & & \\
\hline
\end{tabular}

Abbreviations: OR- odds ratio; 95\% CI-95\% confidence interval. 
Table 4a

Perioperative Outcomes, Morbidity, and Mortality

\begin{tabular}{|c|c|c|c|}
\hline & Epidural (N=120) & No Epidural (N=49) & P-value \\
\hline Days to clear liquid diet & $3.5 \pm 2.6(3,1-25)$ & $3.1 \pm 2.1(2,1-10)$ & 0.07 \\
\hline Days to solid diet & $5.8 \pm 3.3(5,2-28)$ & $7.0 \pm 5.8(5,2-31)$ & 0.58 \\
\hline Length of Stay in days & $11.0 \pm 12.1(8,4-107)$ & $12.2 \pm 10.7(7,3-54)$ & 0.93 \\
\hline TPN use & $9(8 \%)$ & $9(18 \%)$ & 0.053 \\
\hline \multicolumn{4}{|l|}{ Morbidity and Mortality } \\
\hline Pneumonia $^{*}$ & $14(12 \%)$ & $4(8 \%)$ & 0.59 \\
\hline $\mathrm{DVT} / \mathrm{PE}{ }^{* *}$ & $7(6 \%)$ & $2(4 \%)$ & 1 \\
\hline Ileus $q$ I & $16(13 \%)$ & $4(8 \%)$ & 0.44 \\
\hline Pancreatic leak/abscess ${ }^{\dagger}$ & $11(10 \%), \mathrm{N}=107$ & $7(28 \%), \mathrm{N}=25$ & 0.045 \\
\hline Any Morbidity & $43(36 \%)$ & $19(39 \%)$ & 0.73 \\
\hline 30-day Mortality & $5(4 \%)$ & $1(2 \%)$ & 0.67 \\
\hline
\end{tabular}




\section{Table 4b}

Perioperative Outcomes, Morbidity, and Mortality in Patients with Effective Epidurals only vs. No Epidural

\begin{tabular}{|l|l|l|r|}
\hline & Epidural (N=62) & No Epidural (N=49) & P-value \\
\hline Days to clear liquid diet & $3.5 \pm 3.2(3,1-25)$ & $3.1 \pm 2.1(2,1-10)$ & 0.27 \\
\hline Days to solid diet & $5.9 \pm 3.8(5,2-28)$ & $7.0 \pm 5.8(5,2-31)$ & 0.53 \\
\hline Length of Stay in days & $10.3 \pm 9.0(8,4-66)$ & $12.2 \pm 10.7(7,3-54)$ & 0.75 \\
\hline TPN use & $6(10 \%)$ & $9(18 \%)$ & 0.26 \\
\hline Morbidity and Mortality & & & \\
\hline Pneumonia $^{*}$ & $8(13 \%)$ & $4(8 \%)$ & 0.54 \\
\hline DVT/PE ${ }^{*}$ & $2(3 \%)$ & $2(4 \%)$ & 1 \\
\hline Ileus ${ }^{\text {II }}$ & $9(15 \%)$ & $4(8 \%)$ & 0.38 \\
\hline Pancreatic leak/abscess ${ }^{\dagger}$ & $8(14 \%), \mathrm{N}=58$ & $7(28 \%), \mathrm{N}=25$ & 0.13 \\
\hline Any Morbidity & $24(39 \%)$ & $19(39 \%)$ & 1 \\
\hline 30-day Mortality & $1(1.6 \%)$ & $1(2.0 \%)$ & 1 \\
\hline
\end{tabular}

Abbreviations: TPN - Total parenteral nutrition; DVT -Deep vein thrombosis; PE- pulmonary embolism. E- Epidural, NE- No epidural

* Diagnosis determined by an infiltrate on chest X-ray and clinical findings suggesting infection such as purulent secretions, fever, leukocytosis, increasing oxygen requirements, or initiation of antibiotic therapy.

$* *$

Defined based on either chest CT findings or proximal lower extremity thrombosis by duplex ultrasound.

II Defined as nausea, vomiting, or inability to tolerate oral intake by postoperative day 10.

${ }^{\dagger}$ Defined as an intra-abdominal fluid collection requiring drainage and/or any measurable amount of drain fluid after postoperative day 3 with an amylase content greater than 3 times the serum amylase for pancreatic resections. Includes only patients who underwent pancreatic resections. 